\title{
An Objective Infrared Imagery Analysis for Typhoon Jangmi
}

\author{
Chung-Chih Liu and Liang-De Chen
}

\begin{abstract}
A typhoon usually develops from an unstructured cloud cluster. In general, the more axis-symmetry a typhoon cloud pattern is, the stronger the typhoon intensity could be. This study analyzes the MTSAT_IR1 image data of severe typhoon Jangmi during the period from tropical depression to severe typhoon stage. The gradient of the gray values in the MTSAT_IR1 image is calculated from the horizontal and vertical derivatives by using Sobel's template. Then, the deviation angle of each gradient vector relative to a radial line extending from typhoon eye is calculated. The results show that the deviation angle distribution become approaching Gaussian distribution when the intensity of typhoon increase from tropical depression to severe typhoon period, and the structures of cloud cluster will become more axis-symmetry to the center of typhoon.
\end{abstract}

Index Terms-Typhoon jangmi, infrared image, deviation angle.

\section{INTRODUCTION}

Typhoon Jangmi is one of the most intense typhoons during 2008 with a pressure of $905 \mathrm{hPa}$. A tropical disturbance formed in the western Pacific Ocean on September 23, 2008 and then intensified being a typhoon. The intensity of typhoon Jangmi reached its peak wind speeds of 135 kts on September 27. Later that day the outer cloud bands of Jangmi started to interact with the Taiwan's coast. On next day morning, Jangmi made landfall in Yilan County on the east coast of Taiwan.

The physical processes associated with typhoon development have been a subject of considerable interest [1]. Techniques using satellite measurements are perhaps the only way to reliably detect [2]. A combination of visual patterns in the infrared (IR) and visible (VIS) channels brightness temperatures of clouds were usually used to detect rotation, the coldest brightness temperatures are suitable used to detect deep thunderstorms in the eye wall, and the warm brightness temperatures are used to detect an eye at the center of circulation. The cloud pattern of a typhoon associated with its development could be investigated by using satellite remote sensing measurements. It is reasonable to assume that a stronger typhoon has a well organized cloud cluster and an axis-symmetry cloud structure [3], [4]. Accordingly, the organization and axis-symmetry of typhoon cloud increases as the storm develops during the period from tropical depression to typhoon stage. This study calculated and analyzed the deviation angle [4] of infrared image for typhoon Jangmi by using edge image processing technique.

Manuscript received March 12, 1012; revised May 7, 2012.

Authors are with Teaching Center of Natural Science, Minghsin University of Science and Technology, Hsin-Chu county, Taiwan (e-mail: ccliu@must.edu.tw).

\section{DATA}

The satellite data that were used in this study is the Multi-functional Transport Satellite (MTSAT) which is the next generation of GMS- 5 and covers the East Asia and the Western Pacific roughly $35,800 \mathrm{~km}$ above the equator at 140 degrees East longitude. The MTSAT carries one visible and

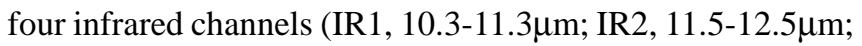

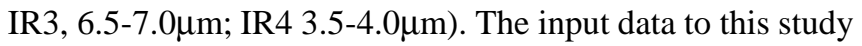
are digital brightness temperatures from long-wave IR1 satellite scenes. The Joint Typhoon Warning Center's (JTWC's) best track data was used in acquiring the position of the typhoon's center hourly by an interpolation technique. In order to concentrate the analysis on typhoon developing period, the data period used in this study is from tropical depression stage to the time point while typhoons arriving the peak wind speeds.

\section{Methodology}

There are so far several different algorithms to be used to examine the meaningful discontinue characteristics in an image. However, the image edge processing technique is the most common. Here, the edge means the region between two sharp distinctive areas. The purpose of edge detecting in the image is to find out the regions with sharp distinctive gray value. Most edge detecting technique includes the partial derivative process. That is, the gradient calculation is usually used in most edge detecting technique.

In general, there is a strong relation between typhoon intensity and structure of typhoon cloud system. Piñeros et al. [4] suggest that the more obvious typhoon circulation cloud band with an axis-symmetry pattern is, the stronger a typhoon is. In this study, the typhoon center was viewed as a reference point and then the position vector of each pixel to the reference point in the image is derived through moving window technique. Also, the gradient vector of each pixel in the same image is derived by using the template factors. Finally, the angle between the position vector and the gradient vector is defined as the deviation angle.

\section{RESULTS}

The JTWC's best track data shows that typhoon Jangmi formed at 12UTC on September 24, 2008 and arrived its peak wind speed at 13UTC on September 27, 2008. The radius of the seventh level of Beaufort wind scale for typhoon Jangmi is $280 \mathrm{~km}$. Therefore, the study calculated and analyzed the deviation angle within this range. The position and maximum wind speed of the typhoon Jangmi was derived hourly by an 
interpolation technique. Then four typhoon stages including tropical depression, mild typhoon, moderate typhoon, and severe typhoon are classified based on the wind speed. Taking the center of typhoon as reference, the correlation between deviation angle and probability density and corresponding gray value image for typhoon Jangmi in the areas were shown in Fig.1.
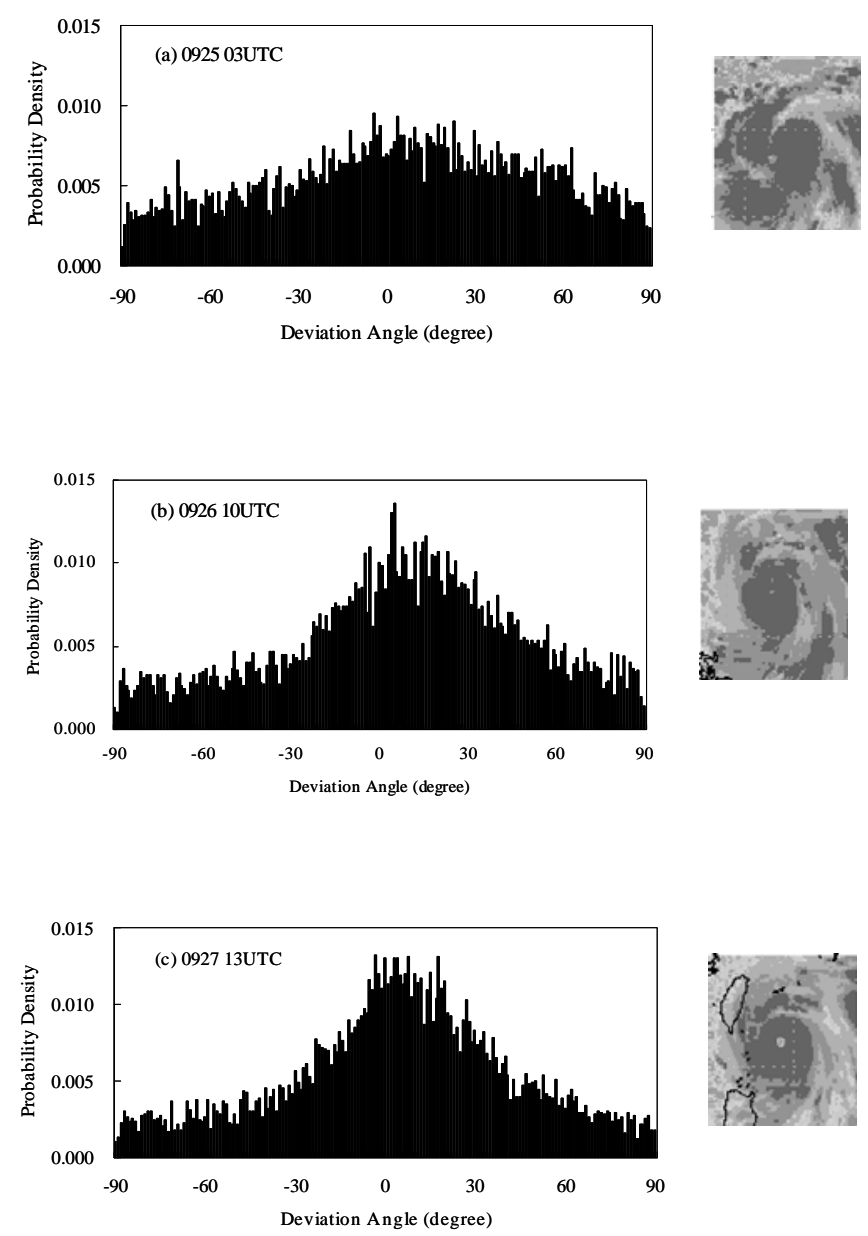

Fig. 1. The correlation between deviation angle and probability density and corresponding gray value image for typhoon Jangmi within $280 \mathrm{~km}$ radius areas about the center of typhoon Jangmi. (a) Mild , (b) Moderate, (c) Severe typhoon stage.

Fig. 1 shows the fact that the stronger the typhoon intensity is, the more axis-symmetric the typhoon cloud pattern and more obvious the gray (or brightness temperature) gradient is Besides, the height of top of the typhoon cloud system is higher for stronger typhoon stage than the weaker typhoon stage. Fig. 2 illustrates the mean correlation between deviation angle and probability density for each typhoon stage. The Fig. 2 shows the results that the stronger typhoon stage has the significant distribution characteristics. That is a larger mean probability density value and smaller standard deviation of deviation angle. Besides, the pattern of the mean correlation between deviation angle and probability density for stronger typhoon stage shows an obvious Gaussian distribution. It also shows a promising application to determine the typhoon density.
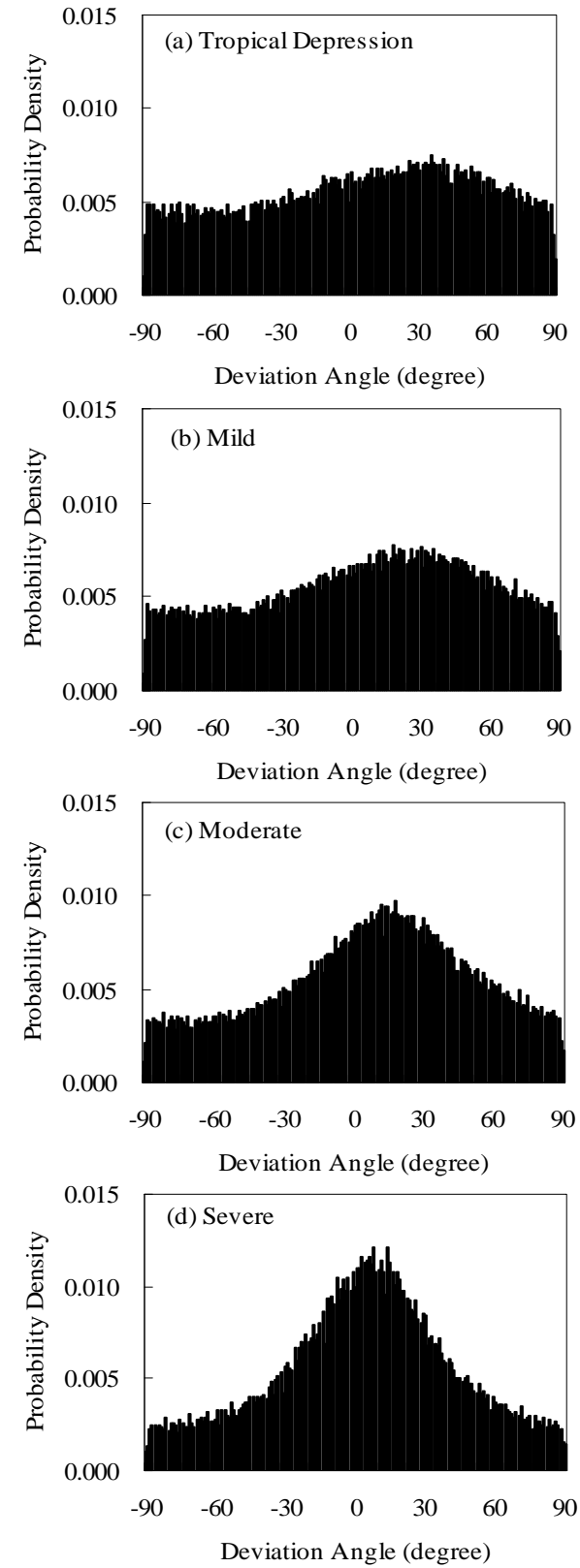

Fig. 2. The mean deviation angle distribution of typhoon during the period in (a)Tropical depression, (b) Mild typhoon, (c)Moderate typhoon, and (d) Severe typhoon stage for Jangmi.

Based on the above results from typhoon Jangmi, the study developed a new indicator (index) in order to quantify the degree of the deviation angle distribution, as expressed in equation 1. Furthermore, the correlation distribution between wind speed and index is shown in Fig.3.

index $=\frac{\left(\begin{array}{l}\text { The probability density } \\ \text { of the peak of distribution }\end{array}\right) \times(90-\text { The degree of the peak })}{90} \times 100$

From Fig. 3, we know the good positive correlation (the R-squared is 0.69) between wind speed and index. It is clearly that the index can characterize efficiently the level of axis-symmetry of the typhoon structure when the intensity increases of typhoon from tropical depression stage. 


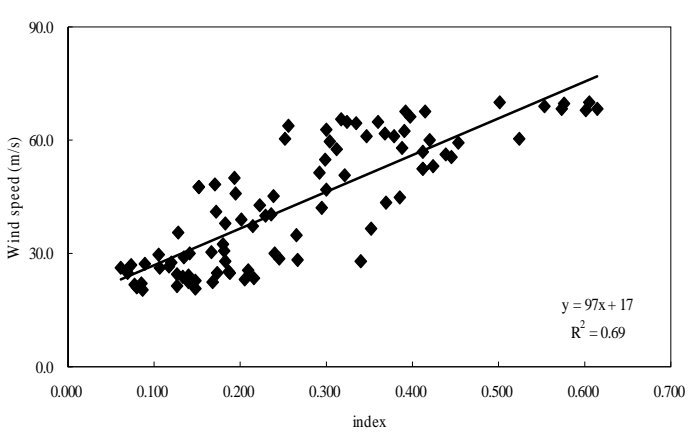

Fig. 3. The correlation distribution between wind speed and index of typhoon from TD to severe stage for Jangmi.

\section{REMARKS}

The results in this study show that the characteristics of typhoon cloud pattern could be displayed through the distribution of deviation angle derived by using image processing technique. The stronger the typhoon intensity is, the more axis-symmetry the typhoon cloud pattern and more obvious the gray (or brightness temperature) gradient is. Besides, the height of top of the typhoon cloud system is higher for stronger typhoon stage than the weaker typhoon stage. It is clearly that the index can characterize efficiently the level of axis-symmetry of the typhoon structure when the intensity increases of typhoon from tropical depression to severe stage. It may be helpful to determine the typhoon intensity.

\section{REFERENCES}

[1] J. McBride, "Tropical cyclone formation. Global view of tropical cyclones,” WMO, Geneva, Switzerland, Tech. Rep, TCP-38, 1995.

[2] P. Wang., P.L. Yang., W.L. Li., H.Q. Lu, "Extracting the rotation feature of the developing typhoon," Proceedings of the Fourth
International Conference on Machine Learning and Cybernetics, Guangzhou, pp. 18-21, August 2005.

[3] M.F. Piñeros., E.A. Ritchie., J.S., Tyo, "Detecting tropical cyclone genesis from remotely sensed infrared image data," IEEE Geoscience and Remote sensing letters, Vol. 7, No. 4, October 2010.

[4] M.F. Piñeros., E.A. Ritchie., J.S., Tyo., Senior Member, IEEE, "Objective measures of tropical cyclone structure and intensity change from remotely sensed infrared image data," IEEE Transactions on Geoscience and Remote sensing, Vol. 46, No. 11, November 2008.

[5] A.E. Dessler., S.P. Palm., J.D. Spinhirne, "Tropical cloud-top height distributions revealed by the Ice, Cloud, and Land Elevation Satellite(ICESat)/Geoscience Laser Altimeter System (GLAS)," Journal of Geophysical Research, vol. 111, D12215, doi:10.1029/2005JF006705, 2006.

[6] C. Casanova., A. Romo., E. Hernández., J.L. Casanova., J. Sanz. "Rapid response for cloud monitoring through Meteosat VIS-IR and NOAA-A/TOVS image function: civil aviation application," A first approach to MSG-SEVIRI. International Journal of Remote Sensing, Vol. 26, Issue 8, 2005

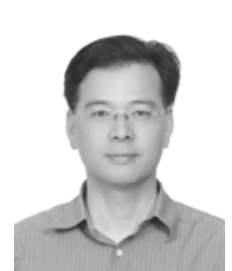

Chung-Chih Liu worked in the teaching center of natural science, Minghsin University of Science and Technology (MUST), Taiwan. He received the B.S degree in 1989 in applied physics from Chung-Cheng Institute of Technology (CCIT), Taiwan, the M.S. degree in 1993 in atmospheric science from National Taiwan University (NTU), Taiwan. Then, he enrolled into National Central University (NCU) and earned the Ph.D. degree in Department of Atmospheric Physics in 2001. His primary research topics are the utilities of satellite data in remote sensing of the physical properties of ocean-atmosphere and their applications on weather forecast.

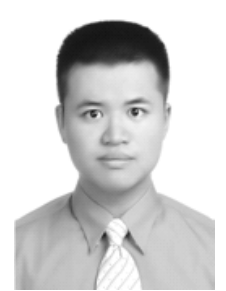

Liang-De Chen worked in the teaching center of natural science, Minghsin University of Science and Technology (MUST), Taiwan. He received the B.S. degree in 2003 in ocean science from National Taiwan Ocean University (NTOU), Taiwan, the M.S. degree in 2005 in marine environmental informatics from National Taiwan Ocean University (NTOU), Taiwan. His primary research topics are the application of satellite data in remote sensing of the physical properties of typhoon. 